\title{
Subcellular Localization of a Novel Transcription Factor in Watered and Drought Sressed Phaseolus acutifolius
}

\author{
L. Rodriguez-Uribe*, S. Ghoshroy**, and M. O’Connell* \\ *Department of Agronomy and Horticulture, ** Electron Microscopy Laboratory, New Mexico State \\ University, Las Cruces, NM 88003 USA
}

Tepary bean (Phaseolus acutifolius) is an agronomically important and drought tolerant legume indigenous to the deserts of Mexico and Southwestern USA. With major droughts and increased competition for water consumption by urban development throughout the world, understanding drought tolerance in plants has become essential. In this study, we investigated the accumulation and subcellular localization of a novel drought-responsive bZIP-like transcription factor in Phaseolus acutifolius. We recently found that transcripts for this bZIP-like trascription factor were specifically accumulated in root in response to drought stress. The complete cDNA sequence for the bZIP from tepary bean was expressed as a recombinant protein in E coli. Protein was purified and used to raise antibodies for use in immunolocalization studies.

Phaseolus acutifolis (O'Odham Brown variety) plants were grown in the greenhouse. Drought stress was applied to an experimental group of plants by withholding water for 7 days. Leaf water potential was measured in both well watered and drought stressed plants to ensure induction of drought. Root samples from well-watered (control) and drought stressed plants were fixed, dehydrated, and embedded in Spurr's epoxy resin. Thick cross sections were made from root tissues to show the general root anatomy. Ultrathin cross sections were made from experimental and control samples. Postsection immunolabeling with anti bZIP antibody followed by $12 \mathrm{~nm}$ colloidal gold conjugated secondary antibody was performed. Subcellular localization of the bZIP was compared between experimental and control samples by visual inspection and by counts of gold particles in standard sized fields.

As expected, the leaf water potential in drought stressed plants was almost two times lower, $-2.1 \mathrm{MPa}$, versus the water potential in well watered plants, -1.1 MPa. Differences in roots samples collected from plants with these leaf water potentials will be the result of the drought stress.

Based on inspection of the immunolabeled TEM images, the bZIP-like protein was detected in epidermal and vascular tissues of the root but not in the cortex or endodermis. There was a three-fold increase in the abundance of this protein in well-watered versus drought-stressed roots. There were two sub-cellular sites for localization, the cell-wall fraction and the nucleus. There was an even staining in the cell walls, for the presence of the bZIP-like protein in control and drought-stressed samples. The differential abundance of the bZIP-like protein appears to be only for the nuclear signal. Within the nucleus, the staining in both control and drought-stressed samples was associated mainly with the heterochromatin fraction.

The surprising observation that the antibody to a bZIP-like transcription factor detects antigen in the cell wall may be explained by a proline rich domain in this transcription factor. We are currently investigating the relationship between transcript levels and protein levels to determine if posttranscriptional or translational regulation is important.

This work has been supported in part by NM AES, USDA CSREES and CONACYT. 


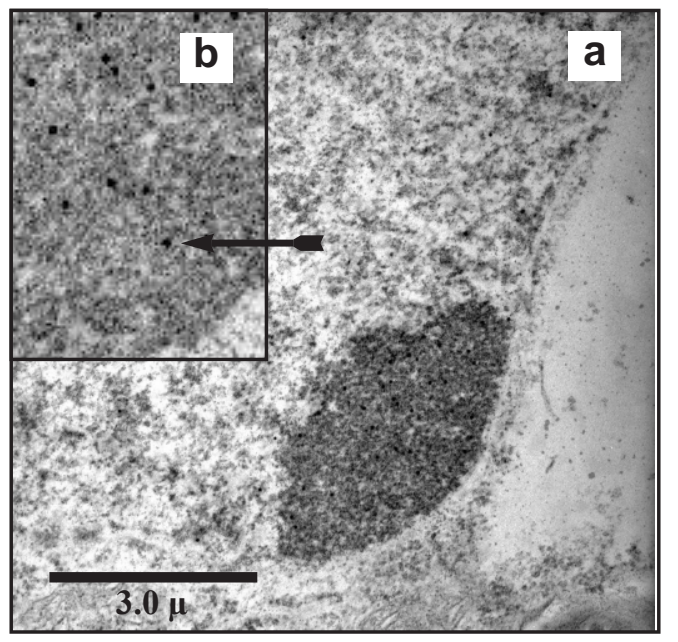

FIG 2a Low magnification TEM of a root epidermal cell from a well watered plant. b High magnification of a root epdermal cell from a well watered plant. Arrow denotes location of gold particle.

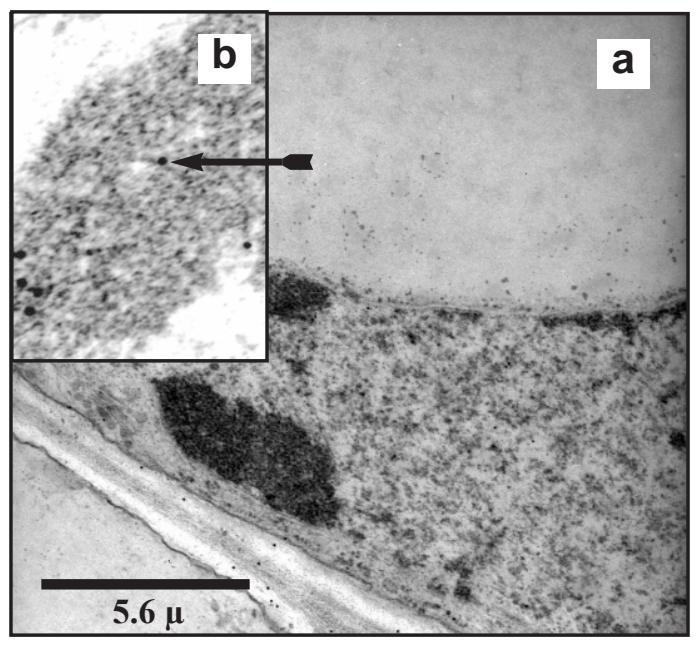

FIG3a Low magnification TEM of a root epidermal cell from a drought stressed plant. b High magnification of a root epdermal cell from a drought stressed plant. Arrow denotes location of gold particle.
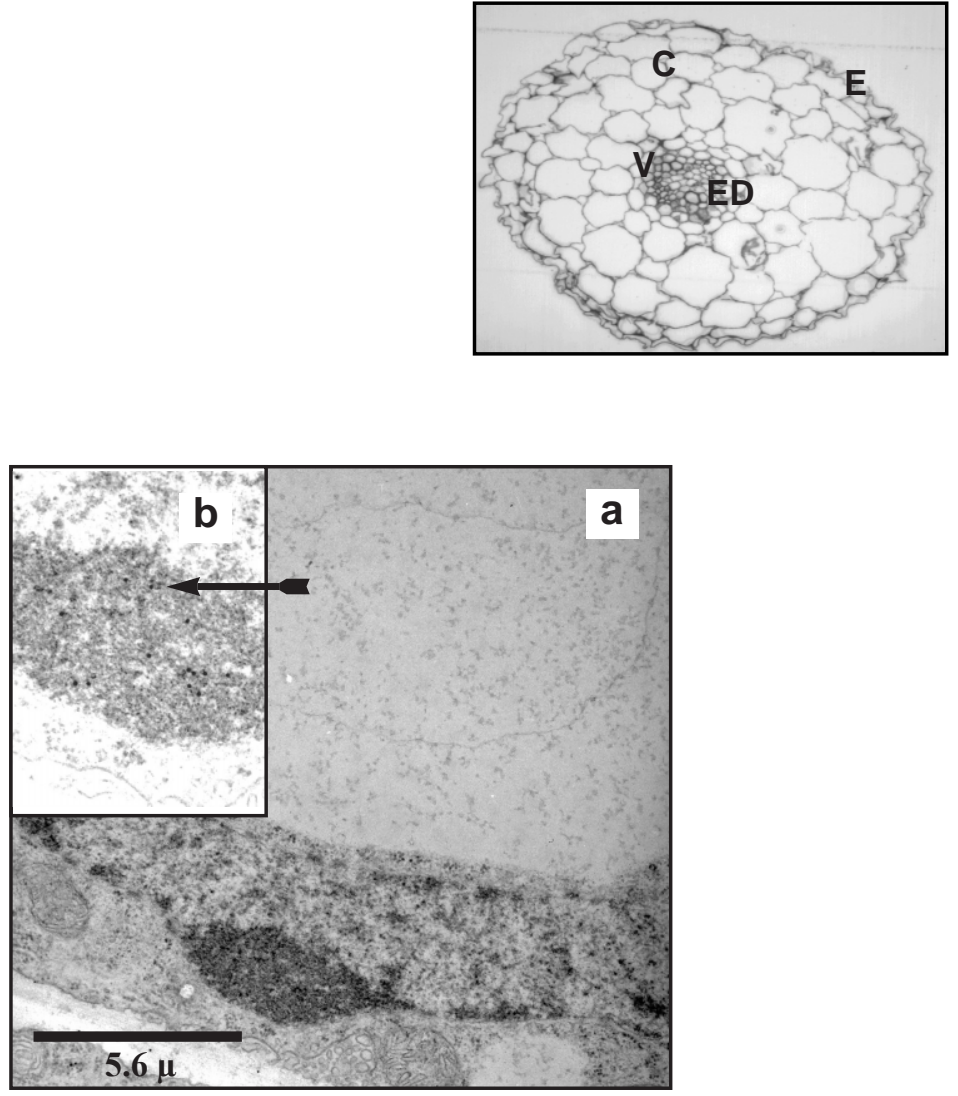

FIG4a Low magnification TEM of a root cortical cell from a well watered plant. b High magnification of a root cortical cell from a well watered plant. Arrow denotes location of gold particle.
FIG 1 Low magnification image from a cross section of a root sample showing the general root anatomy. Epidermis (E), cortex (C), endodermis (ED), vascular (V).

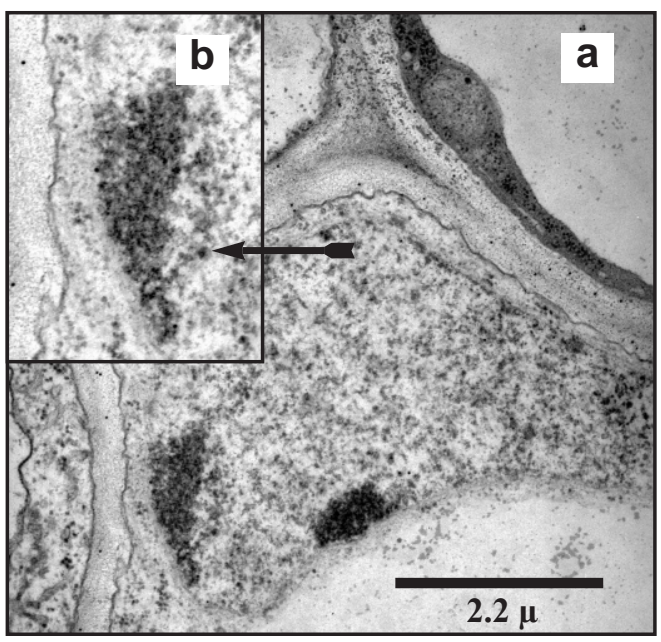

FIG 5a Low magnification TEM of a root cortical cell from a drought stressed plant. b High magnification of a root cortical cell from a drought stressed plant. Arrow denotes location of gold particle. 